\title{
Efficient generation of Bessel beam arrays by means of an SLM
}

\author{
R. Bowman ${ }^{1, a}$, N. Muller ${ }^{2}$, X. Zambrana-Puyalto ${ }^{3}$, O. Jedrkiewicz ${ }^{4}$, P. Di Trapani ${ }^{4}$, \\ and M.J. Padgett ${ }^{1}$ \\ 1 School of Physics \& Astronomy, SUPA, University of Glasgow, Glasgow, Scotland, UK \\ 2 Department of Physics, University of Friburg, 1700 Friburg, Switzerland \\ 3 QISS and Department of Physics \& Astronomy, Macquarie University, 2109 NSW, \\ Australia \\ ${ }^{4}$ CNISM and Università degli Studi dell'Insubria, via Valleggio 11, 22100 Como, Italy
}

\begin{abstract}
We use a Spatial Light Modulator (SLM) to produce arrays of Bessel beams by using multiple axicon phase-masks on the SLM. This approach utilises the whole of the SLM, rather than just a thin annular region (which is the case if the SLM is in the far-field of the generated Bessel beams). Using the whole SLM rather than just an annular region means that the required intensity on the SLM is an order of magnitude lower for a given power in the Bessel beams. Spreading the power over the whole SLM is important for high-power applications such as laser micromachining. We allow the axicons to overlap and interfere in the hologram, so the axial length of the Bessel beam core is maintained as we add more beams to the array.
\end{abstract}

\section{Introduction}

For many applications in modern science and technology it is a requirement to shape the cross section of a laser beam. Such applications range from laser machining and structured light imaging through to the manipulation of microscopic objects in optical tweezers [1-3]. For an obvious route to beam shaping, one need look no further than a standard lecture theatre where the display of the lecture notes uses a data projector. Most data projectors work as pixellated devices where the intensity of the reflected light can be controlled for each pixel, an imaging lens then relays this image to the screen. Complicated, high-resolution, patterns can be created in this way but there are two very obvious drawbacks. Firstly, if the desired pattern is highly localised, e.g. a single small spot, then the process is very inefficient; the spot is formed simply by blocking most of the light. Secondly, if the desired pattern is described not only by the intensity of the light but by its phase as well then an intensity modulation of the projected light gives no obvious mechanism for achieving it. Of course, if the sole objective was to produce a single bright spot of light then this could have been accomplished with high efficiency using a single lens of the appropriate focal length.

${ }^{a}$ e-mail: r.bowman@physics.gla.ac.uk 


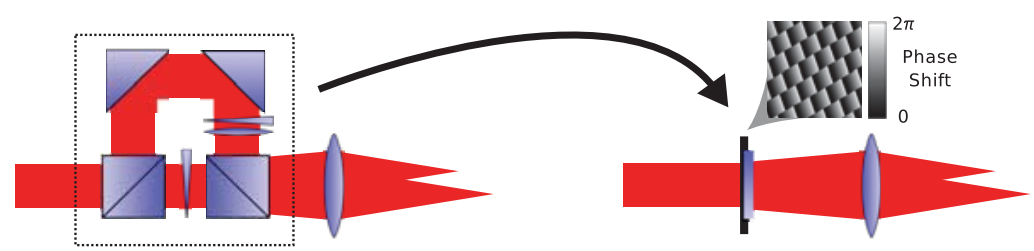

Fig. 1. A holographic element can simulate many optical elements- for example here a hologram (right) is used to independently steer two spots, which would otherwise require a number of components (left).

Rather than create images using selective attenuation, the lens is an example of a component that shapes the phase cross section of the light beam such that, upon propagation, the desired pattern is obtained. Clearly, the incorporation of an additional optical component such as a prism would also allow the focussed light spot to be shifted in a lateral direction. Although a number of beam transformations could be engineered by adding more optical components, this is not a useful approach to general beam shaping. Rather than using refractive optics like lenses and prisms it is possible to perform equivalent transformations using a single diffractive optical element as illustrated in Fig. 1, and described in more detail in [4]. For example, especially for monochromatic light, a lens can be replaced with a phase Fresnel lens (i.e. phase shift $\phi_{\text {lens }} \propto k_{z} r^{2} \bmod 2 \pi$ where $r$ is radial position and $k_{z}$ a constant), and a prism can be replaced by a blazed diffraction grating $\left(\phi_{\text {grating }} \propto k_{x} x \bmod 2 \pi\right.$ where $x$ is position and $k_{x}$ a constant, proportional to the deviation angle). More generally the use of diffraction gratings to shape light beams is called "diffractive optics" or "computer generated holography". Although the study and use of diffractive optics for shaping laser beams dates back many decades it is within the last 10 years that interest in this approach has really exploded.

The dramatic increase in the uses of diffractive optics has been fuelled by two technology advances. Firstly, as we shall see, calculation of the required design of diffraction grating can be complicated and it is only in recent years that such calculations can be completed at a high enough rate to make the process interactive. Secondly, rather than implementing these designs by machining, photolithography, or otherwise, to produce fixed components, a reconfigurable technology has become available. Over the last decade a number of companies have developed technologies for Spatial Light Modulators (SLMs), allowing arbitrary phase patterns to be applied to a light beam. These devices comprise thin liquid crystal cells, where the application of a voltage across the cell introduces a phase delay to the reflected light. Rather than applying the same electric field (and hence the same phase change) across the whole aperture, a desired phase structure can be created by varying the strength of the electric field across the aperture of cell. Most commonly, this spatially varying electric field is created by attaching the liquid crystal cell to a programmable, pixellated CMOS array and using the whole device in reflection mode (which also doubles the available phase shift). Typically, each pixel of the device can create a full $2 \pi$ phase shift with video resolution and update rates. These programmable devices are programmed as secondary monitors from the graphics card of a computer, where an 8-bit greyscale image gives 256 different phase levels.

When programmed in this way, the SLM is effectively acting as a complicated diffraction grating which changes the phase of the reflected light beam such that upon propagation its intensity and phase cross-section transforms into a specific pattern. In essence the SLM is a computer controlled hologram, where the pattern is calculated rather than produced photographically. For simplicity we will now refer to the SLM programmed in this way as the hologram. This approach has a crucial advantage 
over an amplitude modulator such as a digital projector-for an image consisting of a few bright points, the amplitude modulator must block most of the incoming light, and thus achieves poor efficiency. However, the phase modulator can re-direct the incoming light, so that all the available light is sent into the bright spots, thus improving efficiency by an order of magnitude.

Having established that the technology exists whereby an arbitrary phase profile can be imposed upon a reflected light beam, the question remains as to how the required phase profile can be calculated in order to produce a beam which, upon propagation, transforms into the desired shape. Firstly, it is possible to combine simple linear and circular gratings to create laser beams that form an isolated spot or spots in the far field, and these individual spots can be shaped in terms of their intensity and/or phase. This algorithm is often referred to as "gratings and lenses" [2,5].

It is also possible to produce more complicated patterns using iterative Fourier transform algorithms. This approach, first used by Gerchberg and Saxton [6] relies upon the fact that the complex amplitude in a plane is related to that in the far-field of that plane by a Fourier-transform. The intensity and phase structure produced in the far field from any design hologram is obtained by taking the Fourier-transform of the field immediately after the SLM. Conversely, the inverse Fourier-transform maps back from the far-field to the plane of the hologram. By iterating between these two planes, and replacing the intensity (but not the phase) each time with the desired intensity, the hologram will converge on one which produces the desired intensity pattern. However, the effectiveness of this algorithm is greatest when we give it freedom to randomise the phase in the far-field. This is appropriate when generating diffraction-limited spots but not when generating complex beams such as Bessel beams, which have intensity as well as phase structure.

It is also possible to use the SLM in the image plane of the system, converting phase to intensity modulation with Generalised Phase Contrast [7], or to project patterns close to the SLM, in a Fresnel plane [8]. For the generation of our Bessel beams we will work in this Fresnel configuration.

SLMs are also used for tasks other than beam shaping, for example optical image processing $[9,10]$, wavefront measurement and correction [11-14]. This versatility is a key part of the SLM's popularity as a scientific tool.

\section{Creation of a single Bessel beam}

Ideal Bessel beams are infinitely wide, propagation-invariant beams which, for the lowest-order beams, have a bright central core. The finite Bessel beams used in laboratory settings are usually generated with a conical refractive element known as an axicon. The geometry of the Bessel beam generated by an axicon is shown for illustration in Fig. 2 together with its structure in the far field (Fraunhofer) plane. The Fourier transform of a non-diffracting $J_{0}$ Bessel beam corresponds to an infinitely thin ring whose radius $\Theta$ (defined in Fig. 2) is inversely proportional to the size of the central bright spot. However, in the real case, because of the finite size of the input laser beam on the axicon, the resulting far field image of the Bessel beam (more correctly called Bessel-Gauss beam if the illuminating beam is Gaussian) has a well defined width $\Delta \Theta$. Thus, to generate such a beam in the far-field of an SLM, we must display an annular aperture on the modulator.

A set-up with the SLM in the far-field has been used by Cizmar et al. at [15] in order to produce Bessel beams. In their work, a phase mask of the Fourier transform of a Bessel beam is introduced on the SLM where most of the beam is redirected to the zero order, effectively applying a ring shaped amplitude mask. Then, the Bessel beam is obtained by means of a Fourier transform (FT) using a lens. This achieves 

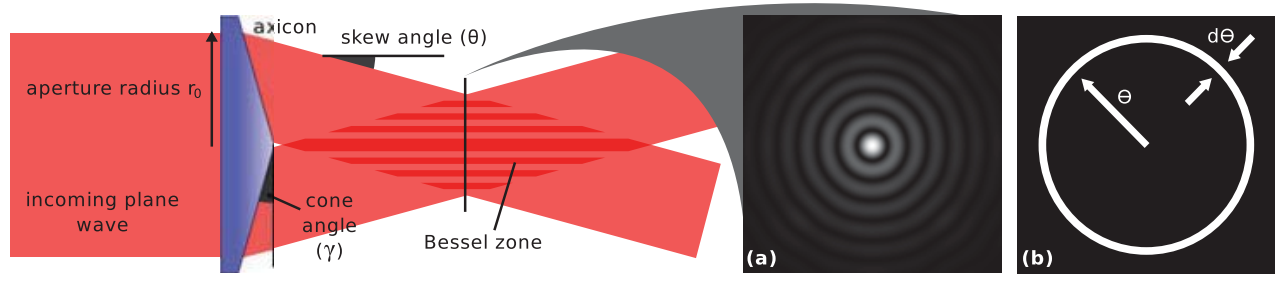

Fig. 2. An axicon produces a Bessel beam when illuminated with a plane wave. Insets show: (a) the Bessel beam, which exists in the "Bessel zone", shaded darker in the figure, and (b) the far-field (Fraunhofer) diffraction pattern.

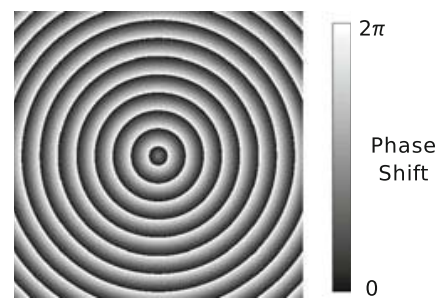

Fig. 3. A hologram corresponding to the phase profile of an axicon produces a Bessel beam close the SLM.

good control over the propagation parameters of the beam, and makes a very pure Bessel mode. However, the annular aperture uses only a part of the incident beam, placing an upper bound on efficiency of order 5\% [15] if a Gaussian beam is used to illuminate the hologram. This is based only on the area of the incident beam which is used, and not on other inefficiencies in the system. It would be possible to improve this using a glass axicon before the SLM to illuminate it with a ring-shaped beam [16]. However this sacrifices much of the flexibility of using an SLM as it fixes the size of the ring $\Theta$ and hence the diameter of the core. We are also left with a very concentrated light pattern on the SLM as the ring has a small area- this would be a problem for laser micromachining applications, where high peak powers are used.

Another way of generating a Bessel beam is to directly mimic a glass axicon on the SLM, as shown in Fig. 3. This produces a Bessel beam which starts immediately after the plane of the SLM, i.e. the Fresnel configuration [8]. For a single beam, this is equivalent to using the SLM as the axicon, and producing a beam as shown in Fig. 2. The holograms have been computed in LabView using the phase profile of an axicon, i.e. $e^{i k \Theta r}$, where $r$ is the radial distance from the centre of the axicon to the respective pixel and $k=2 \pi / \lambda$ is the wavenumber. $\Theta$ is the angle between rays of light in the Bessel beam and the optic axis, related to the cone angle $\gamma$ of the axicon by $\Theta=\left(n_{a x}-1\right) \gamma$ where $n_{a x}$ is the axicon's refractive index. It is possible to add a diffraction grating to this phase structure to move the Bessel beam away from the on-axis undiffracted light, in a similar manner to the "gratings and lenses" algorithm described in [4]. By using the whole incident beam, we would expect to improve the efficiency by at least a factor of ten compared to an annular aperture masking a Gaussian beam.

\section{Creation of an array of Bessel beams}

To produce multiple spots with the SLM in the Fourier plane, we can display a hologram which is the complex superposition of multiple plane waves [3,5,17]. However, 

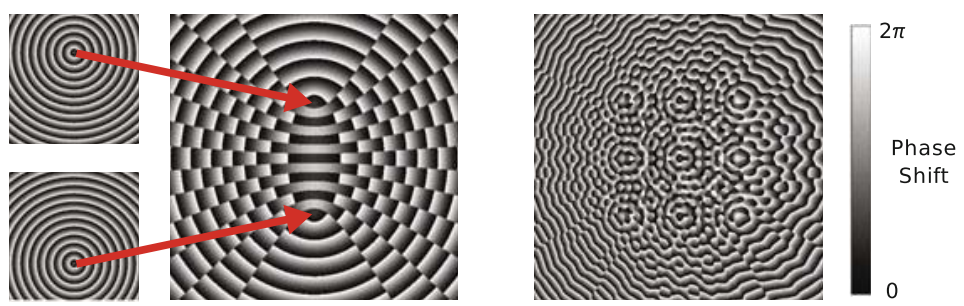

Fig. 4. Holograms for two Bessel beams centred on different parts of the SLM (left) can be combined to produce both beams from a single hologram (centre), by superposing the light fields and extracting the phase. On the right is a hologram for a $3 \times 3$ array of Bessel beams, generated by this method.

with the SLM placed closer to the target plane we must employ a different approachto translate a spot without changing its direction of propagation, we simply translate the corresponding hologram on the SLM, and by including multiple, spatially separate holograms we can create multiple spots [8]. Arrays of spatially separate axicon holograms have been used to generate arrays of Bessel beams [18]. However, the drawback of this approach is that the aperture of each beam is relatively small- for an $n \times n$ array, each spot only uses $1 / n^{2}$ of the available aperture. As the length of a Bessel beam depends on cone angle $\Theta$ and aperture size $r_{0}$, the length $z_{\max }$ of the "non-diffracting" central core of the beams is decreased by $1 / n$.

Our approach draws on both of the above techniques. We work in the Fresnel regime, so as to use the whole area of the SLM, but we superpose the complex fields from the axicons and allow them to overlap as shown in Fig. 4 . This means that $z_{\max }$ no longer decreases as $1 / n$, an advantage that becomes more important as the number of spots in the array increases. It also allows beams to be placed much closer together while maintaining long propagation distances. This helps to avoid non-uniformity in the array due to the illumination becoming less bright near the edge of the hologram.

\section{Experimental setup}

The array of Bessel beams can be generated by summing the complex fields corresponding to the different axicon masks of the array and then extracting the phase of the resulting complex number. The experimental setup used is shown in Fig. 5 . The beam from a He-Ne laser was magnified 20 times by means of a two lens beam expander in order to fill the whole area of the spatial light modulator. The SLM used for the experiment was a PLUTO phase-only modulator (Holoeye, Germany) with a resolution of $1920 \times 1080$ and a pixel pitch of $8 \mu \mathrm{m}$. An imaging telescope (with two lenses of focal length $f_{3}=20 \mathrm{~cm}$ and $f_{4}=40 \mathrm{~cm}$ respectively) was built to image the SLM plane onto a CCD camera, which could be moved on a translation stage along the propagation direction in order to monitor the Bessel beam evolution.

\section{Experimental results}

Figure 6 shows the array of $3 \times 3$ Bessel beams generated as described above, recorded on the CCD camera. The sequence shows the formation of the Bessel beams, how they propagate diffraction free for a distance of about $9 \mathrm{~cm}$ and how they start diffracting again. For the given axicon phase mask used, and considering the parameters of our imaging telescope and the CCD pixels dimensions, we can evaluate that the Bessel 


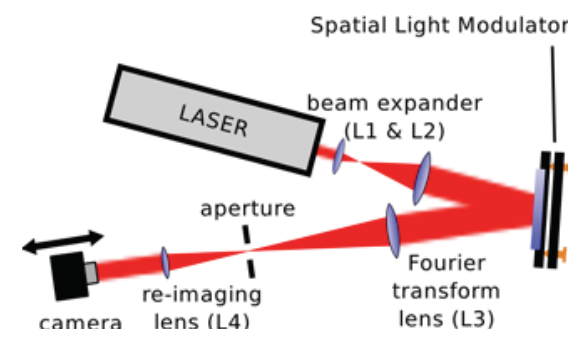

Fig. 5. Experimental setup: the SLM was imaged onto the camera, which was mounted on a translation stage to move along the optic axis. An aperture was placed in the far field of the SLM, between the lenses, to filter out the first order diffraction pattern from the zero order light.
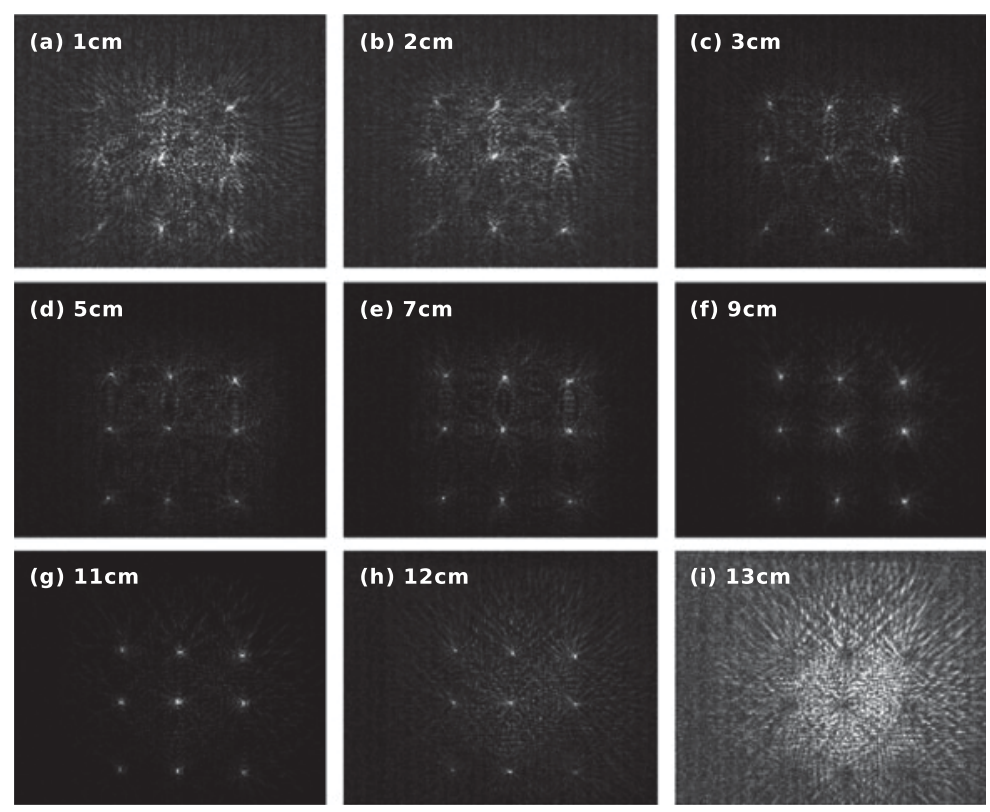

Fig. 6. Transverse section of an array of 9 Bessel beams propagating along the $z$ axis. Positions along the $z$ axis are shown for each image, corresponding to points in Fig. 7.

beams generated and shown in Fig. 6 present a core radius of about $r_{\text {core }} \simeq 42 \mu \mathrm{m}$. Fig. 7 shows the evolution as a function of the propagation distance $z$ of the peak intensity (which was extracted from the maximum intensity recorded by a single CCD pixel) for three different Bessel beams of the array (the Bessel beams positions are illustrated in the inset of the figure).

Although a rigorous approach would have needed the acquisition of different images at the same position (in order to average the peak intensity recorded), and also for many more $z$ positions, the results show a Bessel zone length of $z_{\max } \simeq 9 \mathrm{~cm}$. Note the reduction of the Bessel beams intensity going from the bottom to the top of the array, probably due to a non uniform illumination of the SLM aperture. We expect the fluctuations in intensity in Fig. 7 are artefacts due to the pixellation of the CCD and interference effects from the sensor coverglass- the core size $r_{\text {core }}$ was only a few pixels.

The centres of the Bessel beams are placed in the central $40 \%$ of the SLM, such that the edge of the SLM comes no closer to any given beam's centre than 0.3 times 


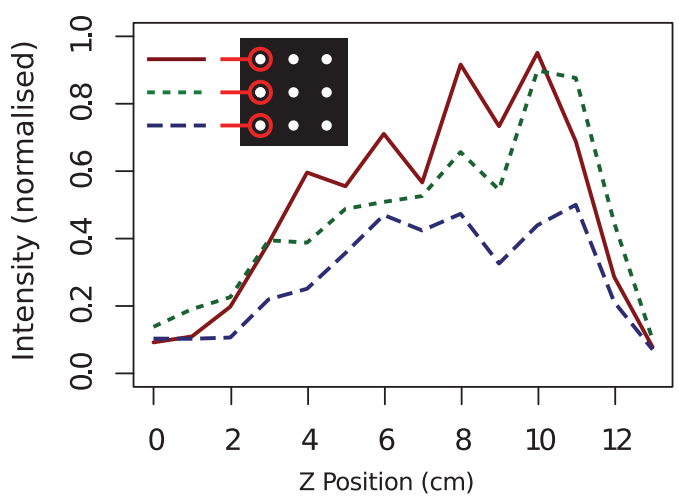

Fig. 7. Recorded peak intensity on the CCD, $I(z)$, for three different Bessel beams in the $9 \times 9$ array generated by means of the axicon phase mask hologram method.

the SLM width. This means that the length of the Bessel zone will be at least 0.6 times that of a single Bessel beam generated with an axicon on the SLM, and usually longer (as the Bessel beam will still be formed after this distance even if part of the aperture is obscured, though its shape will be slightly distorted). This is in contrast to non-overlapping axicons, where three beams would require the length to be reduced by a factor of three compared to the single axicon.

\section{Conclusions}

A new efficient method to generate Bessel beams with an SLM has been presented. This method directly introduces in the SLM the phase variation of an axicon, i.e. $e^{i r \Theta}$. This can be much more efficient in terms of usable power on the SLM, with respect to usual methods of generation where the Fourier transform pattern of the Bessel beam is used as holographic mask for the SLM [15]. Indeed, in the latter case, for the generation of a high quality Bessel beam (long non diffracting propagation distance and narrow core), the ring aperture of the SLM mask must be very thin (ideally the thickness should tend to zero), clearly corresponding to a high power loss and a consequent low efficiency of the generation process. The method proposed here overcomes this limitation. Moreover, it involves the direct use of a single SLM (in contrast to the work of [16], where a real axicon was used in addition to the SLM). Furthermore, we have demonstrated the possibility of efficiently generating arrays of Bessel beams, useful for instance for various applications such as the nano and micromachining of glass channel structures [19] by means of high power pulsed lasers.

The experiment has been performed in the context of STELLA, the School for Training in Experiments with Lasers and Laser Applications, hold at the Insubria University in Como from June 20 to July 8, 2011 (see www.stella-school.eu). The Authors wish to acknowledge CARIPLO, UNIVERCOMO and Banca del Monte di Lombardia Foundations for having financed the project. Authors thank M. Bhuyan, M. Lee and K. Singh for support in the experiment. MJP would like to acknowledge the Royal Society for financial support.

\section{References}

1. A. Ashkin, J.M. Dziedzic, J.E. Bjorkholm, S. Chu, Opt. Lett. 11, 288 (1986)

2. M. Reicherter, T. Haist, E. Wagemann, H. Tiziani, Opt. Lett. 24, 608 (1999) 
3. D.G. Grier, Nature 424, 810 (2003)

4. R.W. Bowman, V. D’Ambrosio, E. Rubino, O. Jedrkiewicz, P. Di Trapani, M.J. Padgett, EPJ (submitted to STELLA school special issue, 2011)

5. J. Liesener, M. Reicherter, T. Haist, H.J. Tiziani, Opt. Commun. 185, 77 (2000)

6. R.W. Gerchberg, W.O. Saxton, Optik 35, 237 (1972)

7. P. Rodrigo, V. Daria, J. Glückstad, Appl. Phys. Lett. 86, 074103 (2005)

8. A. Jesacher, S. Furhapter, S. Bernet, M. Ritsch-Marte, Opt. Express 12, 2243 (2004)

9. S.R.P. Pavani, A. Greengard, R. Piestun, Appl. Phys. Lett. 95, 021103 (2009)

10. C. Maurer, A. Jesacher, S. Bernet, M. Ritsch-Marte, Opt. Express 16, 19821 (2008)

11. L. Seifert, H. Tiziani, W. Osten, Opt. Commun. 245, 255 (2005)

12. I.M. Vellekoop, A.P. Mosk, Phys. Rev. Lett. 101, 12 (2008)

13. T. Cizmar, M. Mazilu, K. Dholakia, Nat. Photon. 4, 388 (2010)

14. R.W. Bowman, A.J. Wright, M.J. Padgett, J. Optics 12, 124004 (2010)

15. T. Čižmár, K. Dholakia, Opt. Express 17, 15558 (2009)

16. T. Cizmar, V. Kollarova, X. Tsampoula, F. Gunn-Moore, W. Sibbett, Z. Bouchal, K. Dholakia, Opt. Express 16, 14024 (2008)

17. J. Leach, K. Wulff, G. Sinclair, P. Jordan, J. Courtial, L. Thomson, G. Gibson, K. Karunwi, J. Cooper, Z.J. Laczik, M. Padgett, Appl. Opt. 45, 897 (2006)

18. S. Tao, X. Yuan, B. Ahluwalia, J. Opt. A-Pure Appl. Opt. 7, 40 (2005)

19. J. Dudley, M. Bhuyan, F. Courvoisier, O. Jedrkiewicz, P. Di Trapani, EPJ (submitted to STELLA school special issue, 2011) 